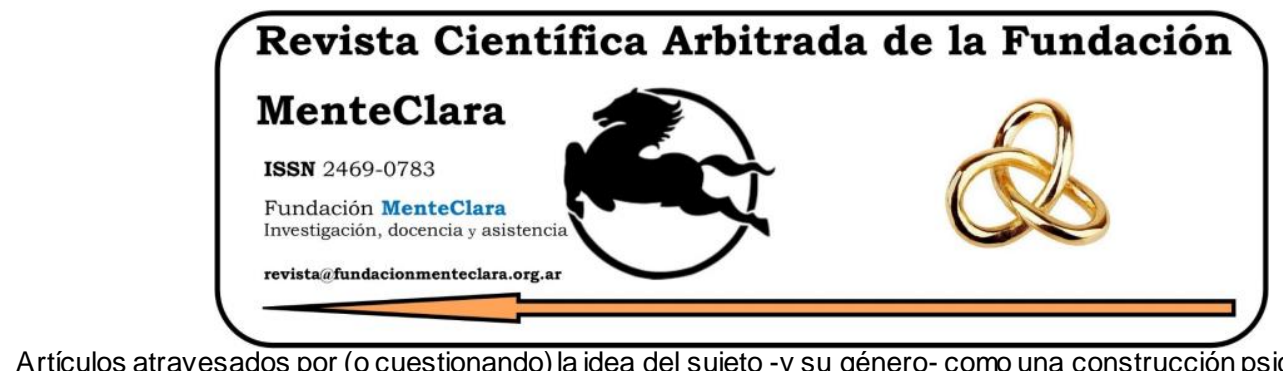

Artículos atravesados por (o cuestionando) la idea del sujeto -y su género- como una construcción psicobiológica de la cultura. Articles driven by (or questioning) the idea of the subject -and their gender- as a cultural psychobiological construction.

Vol. 5 (2020), enero-diciembre

ISSN 2469-0783

https: / / datahub.io/ dataset/2020-5-e154

\title{
¿CÓMO INSTRUMENTAN LOS DOCENTES UNIVERSITARIOS LAS FUNCIONES EJECUTIVAS EN EL EJERCICIO DE SU PROFESIÓN?
}

HOW DO UNIVERSITY TEACHERS INSTRUMENT THE EXECUTIVE FUNCTIONS IN THE EXERCISE OF THEIR PROFESSION?

María Celeste Castelo ${ }^{1}$, Agostina Carbonari2 ${ }^{2}$ Javier Esteban Herrera Galíndez ${ }^{3}$, Candelaria Lestelle ${ }^{4}$

Cómo citar este artículo / Citation: Castelo M. C., Carbonari A., Herrera Galíndez J. E. \& Lestelle C. (2020). "¿Cómo instrumentan los docentes universitarios las funciones ejecutivas en el ejercicio de su profesión?". Revista Científica Arbitrada de la Fundación MenteClara, Vol. 5 (154). DOI: https://doi.org/10.32351/rca.v5.154

Copyright: (C) 2020 RCAFMC. Este artículo de acceso abierto es distribuido bajo los términos de la licencia Creative Commons Attribution 4.0 International License (CC BY 4.0). Recibido: 22/05/2020. Aceptado: 27/05/2020 Publicación online: 28/05/2020

Conflicto de intereses: Ninguno que declarar.

\section{Resumen}

El presente proyecto de investigación corresponde a un estudio descriptivo que tiene por objetivo precisar el uso de las funciones ejecutivas en los docentes de las carreras de Administración de Empresas, Administración Agraria y Abogacía de la Universidad Siglo 21 en la sede de Río Cuarto. La población estuvo integrada por todos los docentes de las carreras anteriormente mencionadas, cuya muestra fue seleccionada a través de un muestreo no probabilístico accidental. Para evaluar las funciones ejecutivas, se utilizó como instrumento el cuestionario autoadministrable "Índice de Funciones Ejecutivas de Jordán”, adecuado anteriormente para tal fin; evaluando atención, inhibición y organización. En esta instancia, se busca llegar a conclusiones sobre los datos recabados que permitan, en un futuro, diseñar un material

\footnotetext{
1 Universidad Siglo 21, Argentina.cele.castelo@hotmail.com

2 Universidad Siglo 21, Argentina. agostina.carbonari018@gmail.com

3 Universidad Siglo 21, Argentina. javierherrera77@hotmail.com

4 Universidad Siglo 21, Argentina.candelest@hotmail.com
} 
audiovisual interactivo con múltiples estrategias que puedan ser implementadas por la universidad en capacitaciones a docentes. Las mismas, tendrán la finalidad de potenciar el uso de estas funciones, mejorando así el rendimiento docente y, por lo tanto, influyendo positivamente en la educación que brinda la universidad local.

\section{Abstract}

This research project corresponds to a descriptive study that aims to specify the use of executive functions in teachers of the careers of Business Administration, Agrarian Administration and Advocacy at the Universidad Siglo 21 at the headquarters of Rio Cuarto. The population was made up of all the teachers of the aforementioned careers, whose sample was selected through accidental non-probability sampling. To evaluate executive functions, the self-administered questionnaire "Jordan Executive Function Index", previously suitable for this purpose, was used as an instrument; evaluating attention, inhibition and organization. In this instance, it seeks to reach conclusions on the data collected that will allow, in the future, to design an interactive audiovisual material with multiple strategies that can be implemented by the university in teacher training. They will have the purpose of promoting the use of these functions, thus improving teaching performance and, therefore, positively influencing the education provided by the local university.

Palabras Claves: Funciones Ejecutivas; Atención; Inhibición; Organización; Docentes Universitarios

Keyw ords: Executive Functions; Attention; Inhibition; Organization; University Teachers 


\section{Introducción}

A partir de los resultados obtenidos en la investigación realizada durante los años 2013-2017, sobre funciones ejecutivas en alumnos pertenecientes a la Universidad Siglo 21 en la sede de Río Cuarto, se observó que al menos el $50 \%$ de los estudiantes poseen algunas dificultades en los componentes de atención, inhibición, planificación y organización del material de estudio. Posteriormente, se indagó acerca de cuáles son las estrategias que utilizan los docentes para desarrollar sus clases, las habilidades que poseen a la hora de planificar las mismas, las modalidades de interacción con los alumnos, los procesos de evaluación, entre otras variables. A partir de los datos recabados, se arribó a la conclusión de que la mayoría de los docentes se desempeñan en su función desde una concepción de enseñanza tradicional.

Dicha modalidad de enseñanza, se caracteriza por una dinámica en la cual el docente es el único poseedor del saber y los estudiantes deben reproducir de manera fiel los contenidos impartidos por el mismo, sin tener en cuenta el componente emocional, los conocimientos previos, los intereses o los factores ambientales. Esta manera de enseñar a partir de la transmisión del conocimiento, dificultaría la construcción del mismo en los estudiantes y, específicamente, la estimulación de funciones cognitivas.

Por ello, se brindaron sugerencias a los docentes con el objetivo de que logren conocer y aplicar nuevas estrategias y metodologias de trabajo para favorecery fortalecer en los alumnos aquellas habilidades en las que se detectaron mayores dificultades. Estas capacitaciones, fueron sustentadas en los conceptos de la Neuroeducación, particularmente sobre las bases neuronales que intervienen en los procesos cognitivos, 
tomando como principios fundamentales a la emoción, atención, novedad, ejercicio físico, juego, arte, sociabilidad y motivación.

En consecuencia, surgieron distintos interrogantes a partir de las inquietudes y la autoevaluación de los docentes en torno a sus propios procesos de pensamiento. Por lo cual, el objetivo es precisar el empleo de las funciones ejecutivas en los docentes de las carreras de Administración de Empresas, Administración Agraria y Abogacía de la Universidad Siglo 21 en la sede de Río Cuarto. Y, puntualmente, se va a describir la utilización de las funciones ejecutivas, analizar su uso y especificar el manejo de la atención, inhibición y organización en dichos docentes.

Para ello, se han tomado las bases de la Neuropsicologia ya que ha contribuido en la explicación de muchas alteraciones o disfunciones que tienen su base en el cerebro e involucran comportamientos que no se pueden analizar sólo desde lo emocional y/o ambiental. En la actualidad, a su vez, esta disciplina ha desarrollado toda una conceptualización en torno a las funciones ejecutivas o habilidades cognoscitivas.

El funcionamiento ejecutivo ha sido estudiado y definido por diferentes autores a lo largo del tiempo (Soprano, 2009) (Rains, 2004) (León-Carrión y Barroso, 1997) (Willis y Mateer, 1992) (Welsh et al, 1991) (Stuss y Benson, 1984) (Luria, 1979).

En este sentido, Pineda las define como un conjunto de habilidades cognoscitivas que permiten la anticipación y el establecimiento de metas, el diseño de planes y programas, el inicio de actividades y operaciones mentales, la autorregulación y monitorización de las tareas, la selección precisa de los comportamientos y conductas, la flexibilidad en el trabajo cognoscitivo y su organización en el tiempo y espacio (Barceló Martínez et al, 2011). 
En esta misma línea, Sholberg y Mateer, en Climent-Martínez et al (2014), consideran que las funciones ejecutivas comprenden una serie de procesos cognitivos entre los que predominan la anticipación, la elección de objetivos, la planificación, la selección de la conducta, la autorregulación, el autocontrol y el uso de realimentación.

En la literatura científica, existe un consenso con respecto a que las funciones ejecutivas se relacionan intrinsecamente con las funciones del Lóbulo Frontal, consideradas como un conjunto de habilidades cognitivas que operan para dar lugar a la consecución de un fin establecido con anticipación (García et al, 2009) (Sánchez Carpintero et al, 2001). Éstas permiten el diseño de planes, la selección de conductas, la autorregulación de los procesos para la consecución del objetivo a realizar, la flexibilidad y la organización de la tarea propuesta. Por lo tanto, se puede observar que las características más relevantes de las mismas son la regulación del self, la secuenciación, la flexibilidad, la respuesta de inhibición, la planificación, la memoria de trabajo y la organización de la conducta.

En relación a lo expresado anteriormente, los estudios más recientes se inclinan por un modelo múltiple, en el que las funciones ejecutivas constituirian la suma de todos los subprocesos requeridos en un determinado momento para una determinada tarea. Los componentes de las funciones ejecutivas son estudiados y especificados por diversos autores, entre ellos Bausela Herrera, en Travaglia et al (2018), quien distingue:

-Inhibición: Es la interrupción de una determinada respuesta que generalmente ha sido automatizada. La estrategia aprendida, debe mantenerse en suspenso ante una nueva situación, permitiendo la ejecución, en ese momento, de otra respuesta. 
-Planificación: Es la capacidad para identificar y organizar una secuencia de eventos con el fin de lograr una meta específica. Para conseguir la meta propuesta, el sujeto debe elaborary poner en marcha un plan estratégicamente organizado de secuencias de acción.

- Flexibilidad: Es la capacidad de alternar distintos criterios de actuación que pueden ser necesarios para responder a las demandas cambiantes de una tarea o situación. Se refiere a la habilidad para cambiar rápidamente de una respuesta a otra, empleando estrategias alternativas.

-Monitorización: Es el proceso que actúa paralela entre a la realización de una actividad. La misma, consiste en la supervisión necesaria para la ejecución adecuada y eficaz de los procedimientos en curso. La monitorización, le permite al sujeto darse cuenta de las posibles desviaciones de su conducta sobre la meta deseada.

-Procesos autoregulatorios: Son los responsables de la organización del comportamiento que permiten, a su vez, la compleja resolución de problemas.

- Memoria de trabajo: También denominada memoria operativa, permite mantener activada una cantidad limitada de información necesaria para guiar la conducta de un sujeto. Por ejemplo, durante el transcurso de una acción, el sujeto necesita disponer de una representación mental tanto del objetivo como de la información relevante.

Los aportes citados anteriormente, permiten un acercamiento a distintos precedentes en relación a la temática. Los mismos, incluyen diferentes conceptos teóricos que se encuentran en íntima relación con la problemática que se aborda en este proyecto de investigación, lo cual demuestra ser de utilidad para este estudio. 


\section{Materiales y Métodos}

Este proyecto de investigación fue de tipo analítico, específicamente descriptivo de tipo transversal.

El método analítico es aquel que consiste en la desmembración de un todo, descomponiéndolo en sus partes o elementos para observar las causas, la naturaleza y los efectos (Ruiz Limón, 2007).

Los estudios descriptivos, buscan especificar las propiedades, las características y los perfiles de personas, grupos, comunidades, procesos, objetos o cualquier otro fenómeno que se someta a un análisis. Las investigaciones trasversales permiten obtener un panorama del estado de una o más variables en uno o más grupos de personas, objetos o indicadores, en un momento determinado (Hernández Sampieri et. al., 2010).

La población estuvo integrada por todos los docentes de las carreras de Licenciatura en Administración de Empresas, Licenciatura en Administración Agrariay Abogacía, que ejercen sus funciones educativas en la Universidad Siglo 21, con sede en la ciudad de Río Cuarto. Dichos docentes fueron seleccionados a partir de los datos recabados en la investigación realizada años atrás, la cual fue referida a las dificultades de los alumnos, y en la que se observó que aquellos que pertenecían a estas carreras presentaban mayores dificultades en el desempeño (Travaglia et. al., 2015).

La muestra, fue un subconjunto de dicha población, la cual estuvo constituida por 34 docentes. La misma, fue seleccionada a través de un tipo de muestreo no probabilístico accidental ya que formaron parte de la muestra aquellos sujetos que respondieron a la encuesta en el periodo establecido en el que se llevó a cabo la recolección de datos. 
La investigación, se implementó siguiendo las pautas éticas de consentimiento informado y anonimato, en el marco de Comités de Éticas Nacionales.

Para evaluar las funciones ejecutivas de dicha muestra, se utilizó un cuestionario de tipo autoadministrable. El instrumento seleccionado, para los propósitos de este estudio, fue el "Índice de Funciones Ejecutivas de Jordán", el cual evalúa atención, inhibición y organización. Este último, es una adaptación realizada para adultos, a través de una valoración inter-jueces en el año 2015 del cuestionario original "Índice de Funciones Ejecutivas para Niños de Jordán” (Travaglia et. al., 2017). Luego de la aplicación del mismo y habiendo analizado los datos recabados, será diseñado un material audiovisual interactivo con múltiples estrategias que puedan ser implementadas por la universidad en capacitaciones a docentes. Las mismas, tendrán la finalidad de potenciar el uso de estas funciones, mejorando así el rendimiento docente $\mathrm{y}$, por lo tanto, influyendo positivamente en la educación que brinda la universidad local.

Para realizar el análisis de los datos cuantitativos, se utilizó el programa estadístico SPSS versión 18. Los principales resultados, se presentan a través de estadísticas, tablas de frecuencias y gráficos representativos, adecuados al tipo de variable en estudio.

Para efectuar el análisis cualitativo de datos, se contempló el Paradigma Interpretativo de Vasilachis (1992). El cual posee, como supuesto básico, que el método para conocer al mundo es la comprensión de las estructuras significativas por medio de la participación en ellas, a fin de recuperar la perspectiva de los participantes y comprender el sentido de la acción en un marco de relaciones intersubjetivas. En este paradigma, las teorias, más que verificarlas o falsearlas con los hechos, 
aparecen como una consecuencia de la comprensión de los mismos. Dicha perspectiva, aportó en la generación de categorías de análisis, emergentes de las voces de los docentes que formaron parte de la muestra.

\section{Resultados}

A partir de las encuestas realizadas, se expondrán los resultados más significativos en torno a las respuestas brindadas por los docentes, en referencia a las funciones ejecutivas evaluadas, específicamente la atención, inhibición y organización.

En cuanto a la atención, se observó que el 58,8\% de los docentes encuestados generalmente mantienen la atención centrada en su trabajo, así como el 29,4\% lo hace siempre y el 11,8\% algunas veces. Los mismos, en su mayoría $(41,2 \%)$ manifestaron que generalmente realizan las tareas necesarias, sin necesidad de recordatorios ni supervisión continúa y un $2,9 \%$ si lo necesita permanentemente. Asimismo, el 50\% de los encuestados recuerdan siempre qué hacer, luego de su labor diaria; sin ser recordado o supervisado; el 44,1\% generalmente; y el 5,9\% algunas veces. También, se expresó un acuerdo (88,3\%) en que los docentes forman parte de algún grupo sin olvidarse del objetivo por el cual se reunieron. Y, en relación con los alumnos, se manifestó que el 85,3\% de los docentes escuchan los aportes de los mismos sin distraerse.

Por otro lado, el 29,4\% de los docentes, afirman que observan siempre los detalles de las cosas, así como el 41,2\% generalmente lo hacen. A su vez, la mayoría de los encuestados (el 50\% siempre y el 26,5\% generalmente) notan cómo se ubican los objetos; el 17,6\% lo hacen algunas veces; en contraposición con el 5,9\% que no lo hace nunca. 
Respecto a la inhibición, en la relación con las demás personas, el $26,5 \%$ de los docentes siempre piensan en las consecuencias de sus actos, el 58,8\% generalmente y el 14,7\% algunas veces. Asimismo, el $8,8 \%$ siempre prioriza las necesidades y el bienestar del otro ante que los propios deseos, el 38,2\% lo hace generalmente y el 52,9\% algunas veces. Se observó, además, que la mayoría de los encuestados (el 20,6\% siempre y el $55,9 \%$ generalmente) tratan de cambiar hábitos y conductas que pueden molestaru ofender a otros; en cambio, el 14,7\% lo realiza algunas veces y el 8,8\% nunca. Del mismo modo, en cuanto a los momentos en los cuales los propios comportamientos dañaron $u$ ofendieron a otros, encontramos en mayor medida $(82,3 \%)$ que los docentes reaccionan ante esta situación ofreciendo disculpas en la totalidad o mayoría de los casos; por otro lado, los demás encuestados $(17,6 \%)$ sólo lo hacen en algunas ocasiones.

Se observó que el 26,5\% de los encuestados siempre dice "no" a sus propios impulsos para llevar a cabo sus responsabilidades y el 29,4\% lo hace generalmente; sin embargo, el 35,3\% lo hace algunas veces y el 8,8\% no lo hace nunca. A su vez, la mayoría de los encuestados (el 35,3\% siempre y el $52 \%$ generalmente) se rigen por las reglas o principios en lugar de actuar de acuerdo a sus impulsos, y el 11,8\% lo hace algunas veces. Cabe destacar, en este sentido, que habria una notable polaridad en cuanto al $50 \%$ de los casos que se orientan a un desempeño flexible y creativo (el 14,7\% siempre y el 35,3\% generalmente), en relación al 50\% restante que responde a una estructuración más rígida (el 44,1\% algunas veces presenta flexibilidad y creatividad, y el 5,9\% nunca lo hace).

En relación a la capacidad de los docentes de establecer objetivos a largo plazo y trabajar sin dejarlos u abandonarlos, se denota que en la mayoría de los casos de manera total $(44,1 \%)$ o en la generalidad $(32,4 \%)$ de las situaciones, logran cumplir con los mismos. Por otro lado, algunos 
docentes refirieron que sólo pueden realizarlos en algunas ocasiones $(20,6 \%)$, mientras que los restantes $(20,6 \%)$ nunca pueden lograrlo.

Y, por último, en relación a la organización, es importante destacar que en la totalidad y generalidad de las veces, la mayoría de los consultados preparan sus horarios $(88,2 \%)$ y recuerdan sus tareas $(91,2 \%)$ sin supervisión; de otro modo, el 11,8\% y el 8,8\% respectivamente lo hacen algunas veces. Por otro lado, el $53,3 \%$ es capaz de escuchar o leer instrucciones siempre, mientras que el 50\% puede hacerlo generalmente y el $14,7 \%$ algunas veces.

En cuanto a la planificación, en vinculación con la distribución del tiempo de manera real sin recibir ayuda, se destaca que el 55,9\% logra hacerlo siempre, el $29,4 \%$ en su generalidad y, por otra parte, el 8,8\% tiene éxito en esta tarea algunas veces, siendo el 5,9\% de los casos aquellos que nunca pueden hacerlo. Dicha planificación es comunicada, por parte de los docentes a otras personas, en la totalidad de las ocasiones por parte del 47,1\% de los educadores; en el mismo porcentaje lo hacen generalmente y el $5,9 \%$ restante lo hace algunas veces.

Otros datos de relevancia, apuntan a la capacidad de mantener los trabajos y apuntes de manera organizada, en este sentido el 88,2\% refieren hacerlo generalmente o en la totalidad de los casos, mientras que el $11,8 \%$ lo hace algunas veces. A su vez, en función a la realización de tareas desde el comienzo hasta el final sin saltarse nada, se evidencia que en la mayoría de los casos se logra de manera total o generalmente $(88,2 \%)$, y el grupo restante $(11,8 \%)$ lo lleva a cabo algunas veces.

\section{Discusión}

Los resultados arrojados del cuestionario evaluado y referidos anteriormente, han podido evidenciar que, en líneas generales, los 
docentes tienen un buen uso de sus funciones ejecutivas. Los datos analizados señalarian que las dificultades de los alumnos se orientarian al modo de transmisión de conocimiento y no exclusivamente al uso de sus funciones ejecutivas.

Se considera central el papel de los docentes como transmisores de conocimiento y responsables en su ética docente en potenciar los procesos de aprendizaje de los alumnos, tomando en cuenta el manejo de sus funciones ejecutivas como eje de gran importancia.

En este punto, adherimos a diversos autores que han apuntado a que existiria un vínculo entre las funciones ejecutivas de los alumnos y el rendimiento de los mismos en actividades de lectura, escritura y cálculo. Actualmente, se expresa un cierto grado de consenso en la comunidad científica, orientado a la presencia de una relación entre el modo de desempeño de las funciones ejecutivas y el rendimiento académico (Stelzer y Cervigni, 2011). Es por ello, que nos orientamos a reforzar y optimizar las mismas en los docentes, a fin de potenciar la manera en la cual transmiten el conocimiento en sus clases. Del mismo modo, se hará hincapié en aquellos docentes con mayores dificultades para que puedan cumplimentar efectivamente las tareas de su rol.

\section{Conclusiones}

A modo de sintesis, es pertinente pensar en la importancia de las funciones ejecutivas en el proceso de enseñanza-aprendizaje. Es por ello que, a partir de los datos recabados a lo largo de las investigaciones realizadas desde 2013 a 2020, se hace evidente que los docentes universitarios tendrían una dificultad en el ejercicio y transmisión de sus propias funciones ejecutivas en relación a los alumnos. Esto se debe a que, como se afirmó anteriormente, los docentes poseen un buen uso de 
sus funciones ejecutivas $\mathrm{y}$, por el contrario, los alumnos tienen dificultades al momento de emplearlas dentro del contexto universitario en el que se encuentran transitando.

Como se puede observar, ambos actores -docentes y alumnospresentan resultados contrapuestos. Por esta razón, se hipotetiza que la causa podría encontrarse en el "entre", es decir, en el lugar intermedio entre ambos. En este caso, se corresponde con la metodología de enseñanza utilizada por los docentes -un modelo de enseñanza tradicional-, ya que es desde la cual se vinculan.

Por lo tanto, se apuntará en la próxima investigación a realizar una correlación entre las funciones ejecutivas y las metodologias de enseñanza utilizadas por parte de los docentes, con la finalidad de comprobar la hipótesis propuesta con anterioridad. 


\section{Referencias}

Barceló Martínez, E., Lewis Harb, S. y Moreno Torres, M. (2011). Funciones Ejecutivas en Estudiantes Universitarios que presentan bajo y alto rendimiento académico. Revista $\mathrm{N}^{\circ}$ 18. Psicología de sde el Caribe. Universidad del Norte.

Climent- Martinez, G., Luna- Lario, P., Bombín- González, I., Cifuentes- Rodriguez, A., Tirapu- Ustárroz, J. y Díaz- Orue ta, U. (2014). Evaluación Neuropsicológica de las funciones ejecutivas me diante realidade virtual. Revista Neurológica $N^{\circ} 58$. Recuperado de: https://www.psyciencia.com/wp-content/uploads/2014/06/Evaluacion-FuncionesEjecutivas-A-Trav\%C3\%83\%C2\%A9s-de-Realidad-Virtual.pdf

García, A., Enseñat, A., Ustárroz, J., Rovira, T. (2009). Maduración de la Corteza Prefrontal y Desarrollo de las Funciones Ejecutivas Durante los Primeros Cinco Años de Vida. Revista de Neurología $N^{\circ} 48$, Supl. 8. (pp. 435-440).

Hernández Sampieri, R., Fernández Collado, C. y Baptista Lucio, M. P. (2010). Me todología de la investigación. Recuperado de: https://www.esup.edu.pe / descargas /dep_investigacion/Metodologia\%20de\%201a\%20in vestigaci\%C3\%B3n\%205ta\%20Edici\%C3\%B3n.pdf

León-Carrión, J. y Barroso, J. M. (1997). Neuropsicología del pensamiento. Control ejecutivo y lóbulo frontal. Kronos: Sevilla.

Luria, A. R. (1979). El cerebro en acción. $2^{\mathrm{a}}$ ed., Barcelona: Fontanella.

Rains, D. (2004). Principios de Neuropsicología Humana. México. Ed. McGraw-Hill.

Ruiz Limón, R. (2007). Historiay Evolución del Pensamiento Científico. Recupe rado de: http: / /www.eumed.net/libros-gratis/2007a/257/index.htm

Sánchez Carpintero, R. y Narbona, J. (2001). Revisión Conceptual del Sistema Ejecutivo y su Estudio en el Niño con Trastorno por Déficit de Atención e Hiperactividad. Revi sta de Neurología $N^{\circ} 33$, Supl. 1. (pp. 47-53).

Soprano, M. (2009). Cómo Evaluar la Atención y las Funciones Ejecutivas en Niños y Adole scentes. Bs. As. Ed. Paidós.

Stelzer, F. y Cervigni, M. A. (2011). Dese mpeño acadé mico y funciones ejecutivas en infancia y adolescencia: una revisión de la literatura. Instituto Rosario de Investigación en Ciencias de la Educación (IRICE-CONICET/UNR).

Stuss, K. H. y Benson, D. F. (1984). Neuropsychological studies of the frontal lobes. Psychological Bulletin. Revista No 95. (pp. 3-28). 
Travaglia, P., Lestelle, C., Rigo, D., Alaminos, C., Meneghello, A., Lorenzoni, M. J., Cervella, S., Castelo, M. C. (2015). Hábitos de estudio y funciones ejecutivas en estudiantes universitarios. Universidad Siglo 21: Argentina.

Travaglia, P., Lestelle, C., Alaminos, C., Cervella, S., Castelo, M. C. (2017). Aprendizaje en la Universidad: ¿Cuáles son los hábitos de estudio que predominan? Universidad Siglo 21: Argentina.

Travaglia, P., Lestelle, C., Alaminos, C., Cervella, S., Castelo, M. C. (2018). La Neuropsicoeducación en la formación doce nte. Universidad Siglo 21: Argentina.

Vasilachis de Gialdino, I. (1992). Métodos Cualitativos I. Los problemas teóricoepiste mológicos. Buenos Aires, Centro Editor de América Latina.

Welsh, H. H., Lind, A. J. y Waters, D. L. (1991). Monitoring Frogs and Toads on Region 5 National Fore sts. As FHR Currents 4. US Fore st Service, Region 5. Eureka, CA. (pp. 12).

Willis, D. y Mateer, C. (1992). Development al impact of frontal lobe injury in kiddle childhood. Special issue: the role of frontal lobe in maturation in cognitive and social development. Brain and Cognition, 20 (1). (pp. 196-204). 